Quim. Nova, Vol. 28, No. 6, 1000-1005, 2005

\title{
DETERMINAÇÃO DE MANGANÊS EM MATERIAL PARTICULADO ATMOSFÉRICO DE AMBIENTES DE TRABALHO UTILIZANDO ELETRODO DE DIAMANTE DOPADO COM BORO E VOLTAMETRIA DE ONDA QUADRADA COM REDISSOLUÇÃO CATÓDICA
}

\author{
Fabiana da Silva Felix, Rita de Cássia Mendes de Barros, Jaim Lichtig e Jorge C. Masini* \\ Instituto de Química, Universidade de São Paulo, CP 26077, 05513-970 São Paulo- SP \\ Neidenêi Gomes Ferreira \\ Instituto Nacional de Pesquisas Espaciais, CP 515, 12210-970 São José dos Campos - SP
}

Recebido em 24/9/04; aceito em 21/3/05; publicado na web em 10/8/05

\begin{abstract}
DETERMINATION OF MANGANESE IN PARTICULATE MATTER OF WORKPLACE ENVIRONMENTS USING A BORONDOPED DIAMOND ELECTRODE AND CATHODIC STRIPPING SQUARE WAVE VOLTAMMETRY. A boron-doped diamond electrode is used for determination of $\mathrm{Mn}$ (II) in atmospheric particulate matter by square wave cathodic stripping voltammetry. The analytical curve was linear for Mn(II) concentrations between 5.0 and $37.5 \mu \mathrm{g} \mathrm{L}^{-1}$, with quantification limit of $3.6 \mu \mathrm{g} \mathrm{L}^{-1}$. The precision was evaluated by the relative standard deviation, with values between $5.1 \%$ and $9.3 \%$. The electrode is free of adsorption, minimizing memory effects. Samples collected in the workplace atmosphere of a foundry had Mn(II) concentrations between 0.4 and $4 \mu \mathrm{g} \mathrm{m}^{-3}$. No significant differences were observed between the proposed method and inductively coupled plasma optical emission spectroscopy.
\end{abstract}

Keywords: manganese; boron-doped diamond; square wave voltammetry.

\section{INTRODUÇÃO}

Fundições são processos industriais muito antigos que datam de aproximadamente 4000 a.C., sendo que os mais usados são a fundição em areia e suas ramificações. Esta é a mais adequada para ferro e aço, com altas temperaturas de fusão, mas também predomina para latão, alumínio, bronze e magnésio ${ }^{1}$. Através do processo de fundição é possível a obtenção de objetos na forma final definida, mediante o vazamento do metal fundido em um molde preparado. A maior parte das empresas da região sul do Brasil utiliza a mesma liga de latão nos processos de fundição de torneiras e registros, composta com aproximadamente $29 \%$ de zinco, $64 \%$ de cobre e o percentual restante constituído de manganês, antimônio, fósforo, enxofre, entre outros ${ }^{2}$.

Considerando que nas etapas do processo ocorre a presença de material particulado na atmosfera do ambiente de trabalho, existe sempre o risco de contaminação do ar, da água, do solo, bem como dos trabalhadores. O manganês é um dos elementos com grande potencial toxicológico, podendo causar danos às funções neurológicas, desordens extrapiramidais, manganismo, bem como bronquite aguda, asma brônquica e pneumonia ${ }^{1,3}$. Métodos muito sensíveis são utilizados para determinação de partículas metálicas no ar, tais como espectrometria de absorção atômica com chama ou forno de grafite, bem como a espectrometria de emissão óptica com plasma acoplado indutivamente (ICP OES) $)^{4-8}$.

Na literatura, são poucas as referências que relatam a determinação de manganês por voltametria de redissolução em particulados atmosféricos ${ }^{9,10}$. Quando aliada à voltametria de onda quadrada ("SWV - Square Wave Voltammetry"), as análises tornam-se mais rápidas e sensíveis, devido, principalmente, à minimização da contribuição da corrente capacitiva, além de se poder trabalhar com altas velocidades de varredura ${ }^{11,12}$. A determinação dos íons $\mathrm{Mn}$ (II) por esta técnica envolve uma etapa de pré-concentração, na qual

*e-mail: jcmasini@iq.usp.br estes íons são oxidados a $\mathrm{Mn}(\mathrm{IV})$, na forma de $\mathrm{MnO}_{2}$, sobre a superfície do eletrodo. Na segunda etapa, há redução da espécie eletroativa oxidada para $\mathrm{Mn}$ (II) mediante uma varredura de potencial no sentido catódico. Filipe e Brett ${ }^{13}$ determinaram manganês em amostras ambientais com eletrodo de carbono obtido a partir de um resistor de $2 \Omega$, com limite de detecção da ordem de $0,22 \mu \mathrm{g} \mathrm{L}^{-1}$. Di e Zhang ${ }^{14}$ obtiveram resultados similares utilizando eletrodo de carbono vítreo. Já Viltchinskaia et al. ${ }^{15}$ e Labuda et al. ${ }^{16}$ reportaram o uso de eletrodos de grafite nas análises de manganês empregando CSV, com limites de detecção da ordem de $0,55 \mu \mathrm{g} \mathrm{L}^{-1}$.

Recentemente novos materiais para eletrodo de trabalho têm sido investigados para aplicações eletroanalíticas, principalmente para evitar o uso de mercúrio (devido a sua toxicidade) ${ }^{17}$. É desejável que tais eletrodos sejam facilmente construídos, apresentem superfícies não suscetíveis a envenenamento ou contaminação, possibilitem análises reprodutíveis, possuam alta seletividade e sensibilidade, apresentem resposta em tempo real, entre outros. O diamante dopado com boro é um destes materiais que, além destas características, apresenta uma ampla janela de potencial de trabalho, baixa corrente de fundo, alta resistência química e estabilidade por longos períodos ${ }^{17-23}$.

O objetivo deste trabalho é a determinação de íons $\mathrm{Mn}$ (II) em amostras de material particulado no ar do ambiente de trabalho de uma fundição de liga de latão empregando Voltametria de Redissolução Catódica por Onda Quadrada ("SWCSV - Square Wave Cathodic Stripping Voltammetry”) com eletrodo de diamante dopado com boro, validando os resultados por comparação com ICP OES, técnica adotada pela "National Institute of Organization Safety (NIOSH)" para análises de metais em amostras de $\operatorname{ar}^{8}$.

\section{PARTE EXPERIMENTAL}

\section{Reagentes e soluções}

Todas as soluções foram preparadas a partir de reagentes de grau analítico da Merck utilizando água ultrapura Milli-Q Plus 
(resistividade de $18 \Omega \mathrm{cm}$ ). Para as análises voltamétricas utilizouse como eletrólito suporte e acerto de $\mathrm{pH}$ a solução de ácido acético/ acetato de amônio $5 \mathrm{~mol} \mathrm{~L}^{-1}$, pH 4,6 e uma solução de $\mathrm{NaOH} 10$ mol L $\mathrm{L}^{-1}$. As soluções analíticas de referência foram preparadas por diluições de uma solução estoque de $\mathrm{Mn}$ (II) $500 \mu \mathrm{g} \mathrm{L}^{-1}$. As soluções analíticas de calibração para as análises por ICP OES foram preparadas a partir de diluições da solução estoque de $\mathrm{MnCl}_{2} 1000$ $\mathrm{mg} \mathrm{L}^{-1}$. Além disso, para as digestões das amostras de filtro de éster-celulose utilizou-se ácido nítrico $10 \%(\mathrm{v} / \mathrm{v})$.

\section{Instrumentação}

As digestões das amostras foram realizadas em forno de microondas fechado CEM MDS 2000, programado para decomposição ácida de óxidos metálicos em uma plataforma giratória de doze vasos de digestão. Os parâmetros utilizados foram $25 \mathrm{~min}$ de tempo de corrida, 80 psi de pressão e $90 \%$ da potência ( $900 \mathrm{~W}$ ).

Os experimentos voltamétricos foram realizados com um potenciostato modelo 263A da EG\&G PARC, tendo eletrodo de diamante dopado com boro como eletrodo de trabalho, eletrodo de referência $\mathrm{Ag} / \mathrm{AgCl}$ ( $\mathrm{KCl}$ saturado) e um fio de platina como eletrodo auxiliar.

As análises empregando ICP OES foram realizadas em um espectrômetro da marca Spectroflame. A tocha estava disposta radialmente e as leituras operaram de modo simultâneo.

\section{Amostragem e preparo das amostras de particulados}

A amostragem foi realizada com bombas individuais portáteis, calibradas para uma vazão de $2,0 \mathrm{~L} \mathrm{~min}^{-1}$. As calibrações da vazão destas bombas de amostragem foram realizadas por calibradores automáticos da marca Gillian, nos quais a vazão média é obtida através do percurso que a bolha de sabão percorre num determinado tempo dentro do próprio calibrador.

Além das bombas, também foram utilizados filtros de éster-celulose da Millipore com diâmetro de $37 \mathrm{~mm}$ e porosidade de $0,8 \mu \mathrm{m}$, que foram montados em porta-filtros de policarbonato da Millipore, compostos de três partes (cassetes) e isolados com fita Teflon ${ }^{\circledR}$. Dois instrumentos de amostragem foram colocados próximos ao forno elétrico da fundição durante dois dias consecutivos do mês de setembro de 2003. As amostragens foram realizadas por um período de $6 \mathrm{~h}$, correspondendo a $70 \%$ da jornada de trabalho de um dado turno de operários da fundição, a qual opera em regime de 24 h. Os cassetes foram mantidos e preservados em caixas metálicas forradas com separadores de espuma até a etapa de digestão.

Após a digestão das amostras com $5 \mathrm{~mL}$ de ácido nítrico concentrado, estas foram transferidas para balões volumétricos de 50,00 $\mathrm{mL}$ com água desionizada, seguindo-se uma filtração lenta, por haver decantação de algumas partículas na forma de sílica ${ }^{8}$. Em seguida, as amostras foram estocadas em frascos de polietileno de $50 \mathrm{~mL}$.

Além da digestão dos filtros, prepararam-se duas amostras em branco do ácido utilizado, bem como duas amostras em branco dos filtros de éster-celulose não utilizados durante as coletas, com o propósito de se verificar possíveis contaminações durante o percurso, ou do próprio filtro empregado.

\section{Preparação e montagem do eletrodo de diamante}

O filme de diamante dopado com boro foi crescido pelo método de deposição química a partir da fase vapor ("Chemical Vapor Deposition - CVD"), o qual emprega temperaturas medianas e pressões sub atmosféricas em reator apropriado ${ }^{24}$.
As condições de obtenção do filme de diamante dopado com boro foram descritas anteriormente ${ }^{25}$, obtendo-se uma taxa de crescimento da ordem de $1 \mu \mathrm{m} \mathrm{h}^{-1}$ e um filme contínuo, sendo que a deposição ocorreu durante $20 \mathrm{~h}$. Na dopagem com boro atingiu-se uma concentração nominal de aproximadamente $10^{21}$ átomos $\mathrm{cm}^{-3}$. Nenhum tratamento posterior do filme foi efetuado antes das análises.

A montagem do eletrodo de diamante iniciou-se pela colagem do filme sobre uma base de cobre, previamente embutida em suporte de Teflon ${ }^{\circledR}$. A colagem tem como objetivo obter o contato elétrico do filme com a base metálica. Neste procedimento, utilizou-se cola condutora de prata. Após um período de espera de aproximadamente 15 min para secagem da cola, aplicou-se, em volta do filme, uma cola de silicone transparente, sempre a partir das bordas do filme em direção ao suporte, de modo que somente o filme de diamante ficasse exposto à solução em estudo. A área superficial resultante foi de $0,14 \mathrm{~cm}^{2}$. Foi possível realizar mais de 200 determinações de Mn por SWCSV para cada montagem do eletrodo.

\section{Determinação de Mn por SWCSV}

Fez-se primeiramente um estudo das melhores condições operacionais para determinação de Mn empregando voltametria. Os parâmetros experimentais obtidos foram potencial de deposição $\left(\mathrm{E}_{\mathrm{d}}\right)=1,2 \mathrm{~V}(v s . \mathrm{Ag} / \mathrm{AgCl})$, tempo de deposição $\left(\mathrm{t}_{\mathrm{d}}\right)=6 \mathrm{~min}$, freqüência $(\mathrm{f})=20 \mathrm{~Hz}$ e altura de pulso $(\Delta \mathrm{E})=25 \mathrm{mV}$.

Para análise de $\mathrm{Mn}$, transferiu-se $40 \mu \mathrm{L}$ do eletrólito suporte ácido acético/acetato de amônio $5 \mathrm{~mol} \mathrm{~L}^{-1}, \mathrm{pH} 4,6$, para uma cela eletroquímica e completou-se o volume com 4,96 mL de água desionizada (prova em branco). Na ausência de nitrogênio e sob agitação, realizou-se a pré-concentração em 1,2 V (vs. $\mathrm{Ag} / \mathrm{AgCl})$ durante $6 \mathrm{~min}$.

Em seguida, realizou-se a varredura de potencial no sentido catódico obtendo-se, assim, as correntes de pico correspondentes ao Mn em determinadas concentrações. Repetiu-se o mesmo procedimento após adição de volumes crescentes de solução padrão de $\mathrm{Mn} 500 \mu \mathrm{g} \mathrm{L}^{-1}$, tanto às provas em branco, como nas soluções provenientes da digestão das amostras de particulados. Para cada determinação, os valores de corrente de pico foram corrigidos em relação às impurezas determinadas nos voltamogramas do filtro em eletrólito suporte ácido acético/acetato de amônio.

\section{RESULTADOS E DISCUSSÃO}

\section{Reações envolvidas e dependência do pH}

A reação para eletrodeposição de $\mathrm{MnO}_{2}$ sobre a superfície de um eletrodo sólido é dada pela Equação 1

$\mathrm{Mn}^{2+}+\mathrm{H}_{2} \mathrm{O} \rightarrow \mathrm{MnO}_{2}+4 \mathrm{H}^{+}+2 \mathrm{e}$

É pouco provável que a reação (1) ocorra em uma única etapa. Um precursor, como o $\mathrm{Mn}^{3+}$, foi sugerido por Rodrigues et al. ${ }^{26} \mathrm{e}$ Petitpierre e Plattner ${ }^{27}$, conforme reação a seguir:

$\mathrm{Mn}^{2+} \rightarrow \mathrm{Mn}^{3+}+\mathrm{e}^{-}$

Os íons Mn(III) podem se desproporcionar em Mn(II) e Mn(IV) em meio ácido, como dado na Equação $3^{28}$, ou podem sofrer hidrólise, formando uma camada intermediária $\mathrm{MnOOH}^{27,29,30}$, de acordo com a Equação 4

$2 \mathrm{Mn}^{3+} \rightarrow \mathrm{Mn}^{2+}+\mathrm{Mn}^{4+}$ 
$\mathrm{Mn}^{3+}+2 \mathrm{H}_{2} \mathrm{O} \rightarrow \mathrm{MnOOH}+3 \mathrm{H}^{+}$

Seqüencialmente, o produto intermediário é oxidado a $\mathrm{MnO}_{2}{ }^{31}$

$\mathrm{MnOOH} \rightarrow \mathrm{MnO}_{2}+\mathrm{H}^{+}+\mathrm{e}$

$\mathrm{O} \mathrm{MnO}_{2}$ pode também ser formado como produto de uma reação de hidrólise ${ }^{29,30}$

$\mathrm{Mn}^{4+}+2 \mathrm{H}_{2} \mathrm{O} \rightarrow \mathrm{MnO}_{2}+4 \mathrm{H}^{+}$

Petitpierre et al. ${ }^{27}$ e Paul e Cartwright ${ }^{29,30}$, a partir de estudos com voltametria cíclica, verificaram que o mecanismo proposto acima segue o modelo de reação do tipo ECE ("ElectrochemicalChemical-Electrochemical") representado pelas Equações 2, 4 e 5. Estes autores também sugeriram que a etapa limitante de todo o processo é a difusão de $\mathrm{Mn}$ (II) através da camada de $\mathrm{MnOOH}$, que possui baixa condutividade elétrica, para junto da superfície do eletrodo. Em altas concentrações de Mn(II), a espessura da camada intermediária é maior e, como consequiência, as reações de oxidação de $\mathrm{Mn}$ (II) para $\mathrm{Mn}$ (III) e para $\mathrm{MnO}_{2}$ são fortemente inibidas. Já em baixas concentrações de Mn(II), há sempre um número de sítios ativos livres de $\mathrm{MnOOH}$ nas quais a reação (2) pode ocorrer.

As etapas eletroquímicas de redução do $\mathrm{MnO}_{2}$ ocorrem conforme Equações 7 e $8^{26,27,32}$

$\mathrm{MnO}_{2}+\mathrm{H}^{+}+\mathrm{e}^{-} \rightarrow \mathrm{MnOOH}$

$\mathrm{MnOOH}+3 \mathrm{H}^{+}+\mathrm{e}^{-} \rightarrow \mathrm{Mn}^{2+}+2 \mathrm{H}_{2} \mathrm{O}$

A estequiometria e a estrutura cristalina do $\mathrm{MnOOH}$, produto da redução do $\mathrm{MnO}_{2}$, são explicadas por algumas teorias, como por ex. a adição de próton mostrada na reação 7 , proposta por Ruetschi ${ }^{33,34}$. Logo, o MnOOH obtido na reação 7 é isomorfo com $\mathrm{MnO}_{2}$. Além disso, Ruetschi ${ }^{33,34}$ sugere que a adsorção e o transporte de íons $\mathrm{H}^{+}$na fase sólida controlam a redução de $\mathrm{MnO}_{2}$.

Há outras reações que podem ser utilizadas na interpretação dos processos que ocorrem com os compostos de manganês. Sabese que $\mathrm{MnOOH}$ é instável em meio ácido, e o processo de desproporcionamento mostrado na Equação 9 acompanha a reação $7^{35}$

$2 \mathrm{MnOOH}+2 \mathrm{H}^{+} \rightarrow \mathrm{MnO}_{2}+\mathrm{Mn}^{2+}+2 \mathrm{H}_{2} \mathrm{O}$

Logo, a reação que corresponde ao processo de redução do $\mathrm{MnO}_{2}$ pode ser descrita pela Equação 10

$\mathrm{MnO}_{2}+4 \mathrm{H}^{+}+2 \mathrm{e}^{-} \rightarrow \mathrm{Mn}^{2+}+2 \mathrm{H}_{2} \mathrm{O}$

Além das reduções eletroquímicas propostas anteriormente ao $\mathrm{MnO}_{2}$, as seguintes reações químicas foram sugeridas por Lee et al. $^{36,37}$

$\mathrm{MnO}_{2}+\mathrm{Mn}^{2+}+2 \mathrm{H}_{2} \mathrm{O} \rightarrow 2 \mathrm{MnOOH}+2 \mathrm{H}^{+}$

$1 / 2 \mathrm{MnO}_{2}+1 / 2 \mathrm{Mn}^{2+}+2 \mathrm{H}^{+} \rightarrow \mathrm{Mn}^{3+}+\mathrm{H}_{2} \mathrm{O}$

$\mathrm{MnOOH}+3 \mathrm{H}^{+} \rightarrow \mathrm{Mn}^{3+}+2 \mathrm{H}_{2} \mathrm{O}$

Os íons Mn(III) gerados nestas etapas químicas podem ser reduzidos eletroquimicamente para $\mathrm{Mn}(\mathrm{II})$, conforme a Equação 14

$\mathrm{Mn}^{3+}+\mathrm{e}^{-} \rightarrow \mathrm{Mn}^{2+}$
No sentido de verificar se a reação representada pela Equação 10 é apropriada para descrever a redissolução do $\mathrm{MnO}_{2}$ acumulado na superfície do eletrodo de diamante, estudou-se a influência do $\mathrm{pH}$ sobre o potencial de redução do $\mathrm{MnO}_{2}$. A partir da reação geral de redução dada na Equação 15 e da Equação de Nernst, obtém-se uma relação linear do potencial de redução em função do pH, cujo coeficiente angular permite estimar o número de elétrons e/ou prótons envolvidos no processo, conforme as reações apresentadas a seguir ${ }^{35}$

$\mathrm{MnO}_{2}+2 \mathrm{e}-+\mathrm{nH}^{+} \rightarrow \mathrm{Mn}^{2+}$

Pela equação de Nernst, tem-se

$\mathrm{E}=\mathrm{E}^{0}-\mathrm{RT} / 2 \mathrm{~F} \ln \left[\mathrm{Mn}^{2+}\right] /\left[\mathrm{H}^{+}\right]^{\mathrm{n}}$

A Equação 16 pode ser rearranjada, fornecendo

$\mathrm{E}=\mathrm{E}^{0}+\mathrm{RTn} / 2 \mathrm{~F} \ln \left[\mathrm{H}^{+}\right]-\mathrm{RT} / 2 \mathrm{~F} \ln \left[\mathrm{Mn}^{2+}\right]$

como

$\mathrm{pH}=-\log \left[\mathrm{H}^{+}\right]=-2,3026 \ln \left[\mathrm{H}^{+}\right]$

Mediante a relação de potencial de redução em função do $\mathrm{pH}$, tem-se

Inclinação $=-2,3026 n R T / 2 F$

A Figura 1 mostra a variação do potencial de redução do Mn(IV) em função do $\mathrm{pH}$, revelando uma relação linear entre os dois parâmetros, com uma inclinação de $-118 \pm 4 \mathrm{mV}$, previsto para 4 prótons $(n=4)$ na Equação 15. Este valor experimental concorda com o valor de $-118,3 \mathrm{mV}$, obtido pela Equação 17 , considerandose $\mathrm{T}=298 \mathrm{~K}, \mathrm{R}=8,31 \mathrm{~J} \mathrm{~K}^{-1} \mathrm{~mol}^{-1}$ (constante dos gases) e $\mathrm{F}=9,65 \mathrm{x}$ $10^{4} \mathrm{C} \mathrm{mol}^{-1}$ (constante de Faraday). As condições experimentais foram as mesmas utilizadas para obtenção da curva de calibração.

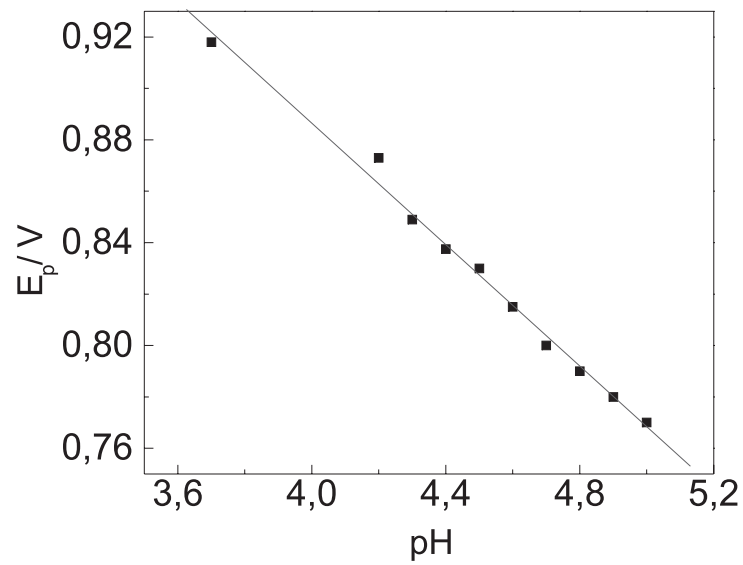

Figura 1. Dependência do potencial de redução do $\mathrm{MnO}_{2}$ em volts (vs. $\mathrm{Ag} / \mathrm{AgCl}$ ) em relação ao pH do eletrólito suporte (ácido acético/acetato de amônio 0,040 mol $\left.L^{-1}\right)$. Condições experimentais: Concentração de $M n(I I)=20 \mu g L^{-1}, E_{d}=$ $+1,2 \mathrm{~V}$ (vs. $\mathrm{Ag} / \mathrm{AgCl}$ ), $t_{d}=6 \mathrm{~min}, f=20 \mathrm{~Hz}, \Delta E=25 \mathrm{mV}, r=0,996$

\section{Características do método analítico}

Para otimização do método, estudou-se inicialmente o potencial de deposição no intervalo de 0,8 a 1,2 V, verificando-se que em 
1,2 V foi obtida a maior relação sinal/ruído para uma solução de $\mathrm{Mn}$ (II) 5,0 $\mu \mathrm{g} \mathrm{L}^{-1}$. O pH foi estudado na faixa entre 3,7 e 5, não se observando uma tendência sistemática. Com isto, adotou-se o valor de 4,6 por se tratar do valor de $\mathrm{pH}$ em que o tampão ácido acético/acetato de sódio apresenta ótima capacidade tamponante. Com relação ao tempo de deposição, o tempo de 6 min permitiu uma boa definição do pico de redissolução catódica para a solução de $\mathrm{Mn}$ (II) 5,0 $\mu \mathrm{g} \mathrm{L}^{-1}$, que corresponderia às soluções de amostras mais diluídas (estudadas previamente por ICP OES).

Utilizando as condições experimentais otimizadas, ou seja, potencial de deposição $\left(\mathrm{E}_{\mathrm{d}}\right)=1,2 \mathrm{~V}(v s . \mathrm{Ag} / \mathrm{AgCl})$, tempo de deposição $\left(\mathrm{t}_{\mathrm{d}}\right)=6 \mathrm{~min}$, frequiência $(\mathrm{f})=20 \mathrm{~Hz}$ e altura de pulso $(\Delta \mathrm{E})=25 \mathrm{mV}$, construiu-se a curva analítica mostrada na Figura 2. Para uma faixa de concentrações entre 5,0 e $40 \mu \mathrm{g} \mathrm{L}^{-1}$ de $\mathrm{Mn}(\mathrm{II})$, pode-se verificar uma relação linear entre corrente de pico e concentração do analito, resultando na equação $i_{p}=(11,0 \pm 0,2)\left[\mathrm{Mn}^{2+}\right]-(6 \pm 4)$, com $\mathrm{r}=$ 0,998 e coeficiente angular dado em unidades de $\mathrm{nA} \mathrm{L} \mu \mathrm{g}^{-1}$.

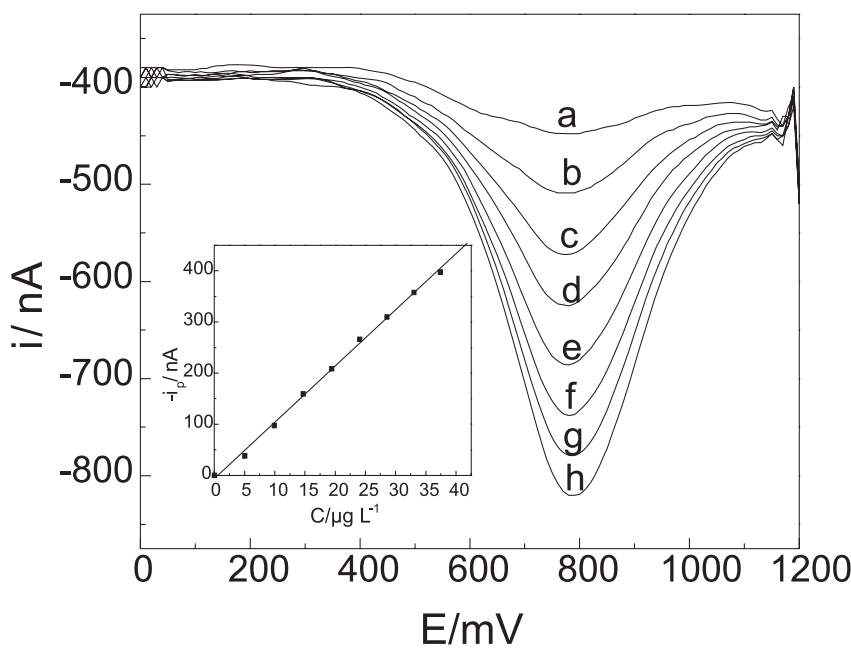

Figura 2. Voltamogramas de redissolução catódica por onda quadrada para soluções de Mn(II) de concentrações: (a) 5,0 , (b) 9,90, (c) 14,71, (d) 19,42,

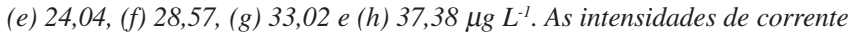
referem-se à resultante dos pulsos diretos e reversos. Os experimentos foram realizados em meio de tampão ácido acético/acetato de amônio 0,040 mol $\mathrm{L}^{-1}$ ( $\mathrm{pH} 4,60)$, com as demais condições experimentais descritas na legenda da Figura 1. A figura inserida corresponde à curva analítica, descrita pela equação $i_{p}=(11,0 \pm 0,2)[\mathrm{Mn}(I I)]-(6 \pm 4)$, com $r=0,998$

O valor de Limite de Quantificação $(L Q)$ obtido por SWCSV foi calculado, em $\mu \mathrm{g} \mathrm{L} \mathrm{L}^{-1}$, a partir da expressão: $L Q=10 \mathrm{~S}_{b} / \mathrm{m}$, onde $S_{b}$ corresponde à estimativa do desvio padrão do branco e $m$ à inclinação da curva analítica, com 11 leituras. O valor de Limite de Detecção $(L D)$ foi obtido utilizando-se a expressão $L D=3 S_{b} / \mathrm{m}^{38}$. Os resultados foram $L D=1,0 \mu \mathrm{g} \mathrm{L}^{-1}$ e $L Q=3,6 \mu \mathrm{g} \mathrm{L}^{-1}$. Os valores de $\mathrm{LD}$ e $\mathrm{LQ}$ obtidos foram apropriados para determinação de $\mathrm{Mn}$ (II) em particulados atmosféricos coletados em fundições. Tais limites podem ser sensivelmente melhorados aumentando-se a eficiência do transporte de massa durante as etapas de acumulação e redissolução, conforme demonstrado por Saterlay et al..$^{35}$ que utilizaram um sistema de ultra-som para atingir limites de detecção da ordem de $10^{-11} \mathrm{~mol} \mathrm{~L}^{-1}$.

Os valores de $L D$ e $L Q$ para ICP OES foram calculados como sendo a concentração, em $\mu \mathrm{g} \mathrm{mL}{ }^{-1}$, correspondente a 3 e 10 vezes o valor da estimativa do desvio padrão do branco com 11 leituras, respectivamente $^{38}$. Estes valores foram $L D=0,70 \mu \mathrm{g} \mathrm{L}^{-1}$ e $L Q=2,3$ $\mu \mathrm{g} \mathrm{L}^{-1}$, valores estes bem próximos daqueles obtidos por SWCSV.

O método eletroquímico apresentou desvios padrões relativos que variaram entre 5,1 e 9,3\% para amostras com concentrações entre 6,4 e $14,4 \mu \mathrm{g} \mathrm{L}^{-1}$ (Tabela 1). Esta boa precisão pode ser atribuída à ausência de processos de adsorção irreversíveis sobre a superfície do eletrodo de diamante dopado com boro, aliada ao tempo de acumulação relativamente longo utilizado.

Tabela 1. Comparação das médias das concentrações de Mn(II) das análises por SWCSV com os valores obtidos por ICP OES, em $\mu \mathrm{g} \mathrm{L^{-1 }}$

\begin{tabular}{lccccc}
\hline \multirow{2}{*}{ Amostra } & \multicolumn{2}{c}{ ICP OES } & \multicolumn{2}{c}{ Voltametria de } & \multicolumn{2}{c}{ Redissolução } & $\mathrm{t}$ \\
& $\mathrm{M}_{1}$ & $\mathrm{DP}$ & $\mathrm{M}_{2}$ & $\mathrm{DP}$ & \\
\hline 1 & 8,2 & 0,9 & 10,8 & 0,8 & 1,63 \\
2 & 9 & 4 & 13,5 & 0,7 & \\
3 & 12 & 3 & 14,4 & 0,9 & \\
4 & 8 & 1 & 6,4 & 0,6 & \\
\hline
\end{tabular}

$\mathrm{M}=$ Média aritmética, DP = Estimativa do desvio padrão, $\mathrm{t}=\left(\mathrm{M}_{1}\right.$ $\left.-\mathrm{M}_{2}\right) \sqrt{ } \mathrm{n} / \mathrm{DP}$ com $\mathrm{n}=$ número de determinações $(\mathrm{n}=4)$. $\mathrm{O}$ valor crítico de té 3,18 para um nível de significância de 0,05 e para três graus de liberdade ${ }^{38}$.

\section{Análises das amostras e avaliação ambiental}

A quantificação de Mn(II) em amostras de digeridos de material particulado atmosférico coletados na fundição em estudo foi efetuada pelo método de adições de padrão, sendo os resultados apresentados na Tabela 1. Para verificar se a metodologia proposta para a determinação de $\mathrm{Mn}$ em amostras de ar é compatível com aquela reconhecida pela $\mathrm{NIOSH}^{8}$, que recomenda a utilização do ICP OES nas determinações, aplicou-se o teste estatístico t-Student pareado para comparação da média experimental com o valor obtido por ICP OES, conforme mostrado na Tabela 1. Como o valor crítico de t é igual a 3,18 para um nível de significância de 0,05 e para três graus de liberdade ${ }^{38}$ e o valor de t obtido na Tabela 1 é menor que este valor, conclui-se que não há evidência de diferenças estatisticamente significativas entre os valores comparados.

Os resultados apresentados na Tabela 1 foram apropriadamente corrigidos em função da vazão fornecida pela bomba, assim como pelo tempo de amostragem, de modo a fornecer valores em $\mu \mathrm{g}$ de $\mathrm{Mn}$ por $\mathrm{m}^{3}$ de ar. Estes valores foram da ordem de $0,4 \mathrm{a} 4 \mu \mathrm{g} \mathrm{m}^{-3}$, estando bem abaixo do Limite de Concentração de $200 \mu \mathrm{g} \mathrm{m}^{-3}$ recomendado pela "American Conference of Governmental Industrial Hygienists (ACGIH)", de modo que se pode inferir que os trabalhadores desta fundição não estão sendo expostos a concentrações de $\mathrm{Mn}$ prejudiciais à saúde. Por outro lado, um estudo recente demonstrou que as concentrações de $\mathrm{Cd}$ e $\mathrm{Pb}$ nestas atmosferas são sistematicamente maiores que os Limites de Concentração recomendados pela $\mathrm{ACGIH}^{39,40}$.

\section{Interferências}

Como a matriz dos particulados apresenta uma grande diversidade de íons presentes, realizou-se um estudo sobre possíveis interferentes nas determinações voltamétricas. $\mathrm{O}$ critério utilizado para escolha dos íons interferentes está no fato de que os íons $\mathrm{Cu}$ (II) e Zn(II) são os elementos em maior proporção na liga de latão, bem como os íons $\mathrm{Cd}(\mathrm{II}), \mathrm{Pb}(\mathrm{II})$ e $\mathrm{Ni}$ (II) são prováveis íons da liga de latão que apresentam um grande potencial toxicológico. A escolha da concentração do metal de interesse localizou-se em um dos primeiros pontos da curva analítica, bem como pelo fato do pico de redissolução apresentar-se bem definido.

Considerou-se o íon testado como interferente quando houve 
uma variação em $\pm 5 \%$ na corrente de pico do metal selecionado, à medida que o íon era acrescentado à solução. Para tanto, utilizouse uma solução de $\mathrm{Mn}$ (II) 5,0 $\mu \mathrm{g} \mathrm{L}^{-1}$ em eletrólito suporte ácido acético/acetato de amônio $0,040 \mathrm{~mol} \mathrm{~L}^{-1}$ em presença de $\mathrm{Cd}(\mathrm{II})$, $\mathrm{Cu}(\mathrm{II}), \mathrm{Ni}(\mathrm{II})$ e $\mathrm{Zn}(\mathrm{II})$ nas concentrações de 20,0 e $570 \mu \mathrm{g} \mathrm{L}^{-1}$. A interferência de $\mathrm{Pb}(\mathrm{II})$ foi estudada com soluções deste íon em concentrações 5 e $305 \mu \mathrm{g} \mathrm{L}^{-1}$, obtendo-se as razões de concentração entre os metais de interesse e os prováveis íons interferentes mostrados na Tabela 2.

Tabela 2. Influência dos prováveis íons interferentes sobre a corrente de pico de redissolução para soluções de $\mathrm{Mn}$ (II) 5,0 $\mu \mathrm{g} \mathrm{L}^{-1}$ na ausência e presença de nitrato de sódio

\begin{tabular}{lccc}
\hline Interferente & Razão $\mathrm{C}_{\text {interf }} / \mathrm{C}_{\mathrm{Mn}(\mathrm{II})}$ & $\Delta \mathrm{Ip}(\%)^{\mathrm{a}}$ & $\Delta \mathrm{Ip}(\%)^{\mathrm{b}}$ \\
\hline $\mathrm{Cd}(\mathrm{II})$ & 4 & $* *$ & $* *$ \\
& 114 & $-6,4$ & $+8,1$ \\
$\mathrm{Cu}(\mathrm{II})$ & 4 & $-2,2$ & $-1,4$ \\
& 114 & $-69,1$ & $-4,4$ \\
$\mathrm{Ni}(\mathrm{II})$ & 4 & $* *$ & $+2,3$ \\
& 114 & $>-100$ & $-29,4$ \\
$\mathrm{~Pb}(\mathrm{II})$ & 1 & $-2,4$ & $* *$ \\
$\mathrm{Zn}(\mathrm{II})$ & 61 & $-59,4$ & $-2,8$ \\
& 4 & $-4,2$ & $+2,9$ \\
& 114 & $-23,1$ & $+4,0$ \\
\hline
\end{tabular}

onde $\mathrm{C}_{\text {interf }}=$ Concentração do provável íon interferente, $\mathrm{C}_{\mathrm{Mn}(\mathrm{II})}=$ Concentração do íon $\mathrm{Mn}(\mathrm{II})$ e $\Delta \mathrm{Ip}=$ Variação na corrente de pico do íon $\mathrm{Mn}(\mathrm{II})$, ** corresponde a uma variação desprezível. a) ausência de nitrato de sódio; b) presença de nitrato de sódio.

A partir dos valores de $\Delta \mathrm{Ip}(\%)$ mostrados na Tabela 2, observase que nas soluções em que as concentrações dos possíveis interferentes excedem em 100 vezes a concentração do íon $\mathrm{Mn}$ (II) há interferências que provocam a diminuição da corrente de pico para a maioria dos íons selecionados. Porém, para o íon $\mathrm{Pb}(\mathrm{II})$ a interferência negativa manifesta-se mais pronunciadamente quando sua concentração excede em 61 vezes a concentração do íon Mn(II). Como as amostras de particulados no ar sofreram adições de hidróxido de sódio $10 \mathrm{~mol} \mathrm{~L}^{-1}$ para diminuir a acidez do meio, a qual é decorrente da etapa de digestão, fez-se um novo estudo de possíveis interferentes para $\mathrm{Mn}$, obtendo-se os valores descritos na segunda coluna de $\Delta \mathrm{Ip}(\%)$ da Tabela 2. Para tanto, utilizou-se uma solução de $\mathrm{Mn}(\mathrm{II})$ $5,0 \mu \mathrm{g} \mathrm{L}^{-1}$ em nitrato de sódio $2 \mathrm{~mol} \mathrm{~L}^{-1}$, além do eletrólito suporte ácido acético/acetato de amônio $0,040 \mathrm{~mol} \mathrm{~L}^{-1}$. Os resultados de $\Delta \mathrm{Ip}(\%)$ mostram um comportamento bem diferente do Mn na presença dos possíveis interferentes em meio de nitrato de sódio. Não há mais uma diminuição generalizada na corrente de pico, assim como valores altos de $\Delta \mathrm{Ip}$. A maioria das interferências ocorre quando os íons selecionados excedem em 100 vezes a concentração do íon $\mathrm{Mn}(\mathrm{II})$, como por ex., em adições do íon $\mathrm{Ni}$ (II) que, sob estas condições, diminui o sinal da corrente de pico do íon de interesse, mas não o suprime completamente, como acontece na ausência de nitrato de sódio. A presença do íon $\mathrm{Pb}(\mathrm{II})$ em concentrações excedendo em até 61 vezes a concentração do íon $\mathrm{Mn}$ (II) não interfere mais. Entretanto, um aumento na razão $\mathrm{Pb} / \mathrm{Mn}$ leva a um aumento na corrente de pico, bem como o potencial de redução desloca-se para valores mais negativos, em concordância com as observações feitas por Jin et al. ${ }^{41}$ e Filipe et al. ${ }^{13}$. Esta interferência é provavelmente devida ao fato de que os íons $\mathrm{Pb}$ (II) são facilmente oxidados, com formação de $\mathrm{PbO}_{2}$, o qual pode ser depositado sobre a superfície do eletrodo de trabalho juntamente com o óxido de manganês.

\section{CONCLUSÕES}

O eletrodo de diamante dopado com boro mostrou bom desempenho, podendo ser utilizado como eletrodo de trabalho nas determinações de Mn em material particulado atmosférico utilizando a técnica de SWCSV. O método proposto apresentou limites de detecção e quantificação da mesma ordem de grandeza que os obtidos por ICP OES, sendo que os resultados das concentrações de Mn no material particulado atmosférico não apresentaram evidências de diferenças estatisticamente significativas entre os dois métodos. Dentre as propriedades do eletrodo de trabalho, pode-se destacar a ausência de efeitos de adsorção, uma característica extremamente vantajosa do filme de diamante dopado como boro para fins analíticos, uma vez que minimiza os efeitos de memória. Além disso, pôde-se verificar uma corrente de fundo baixa e estável, o que acarreta uma elevada relação sinal/ruído para vários sistemas redox, o que também é interessante do ponto de vista analítico devido à alta sensibilidade das medidas.

\section{AGRADECIMENTOS}

À FAPESP, ao CNPq e à CAPES pelo apoio financeiro, assim como à FUNDACENTRO por viabilizar a coleta das amostras.

\section{REFERENCIAS}

1. Stellman, J. M.; Encyclopedia of Occupational Health and Safety, $4^{\text {th }}$ ed., International Labour Office: Geneva, 1998.

2. FUNDACENTRO, Fundação Jorge Duprat Figueiredo de Segurança e Medicina do Trabalho; Relatório de Fundições, São Paulo; 1999.

3. Oga, S.; Fundamentos de Toxicologia, Ed. Atheneu: São Paulo, 1996.

4. Moreira, F.; Pivetta, F.; At. Spectrosc. 1998, 19, 137.

5. Lee, Y.; Smith, M. V.; Indurthy, S., Deval, A; Sneddon, J.; Spectrochim. Acta, Part B 1996, 51, 109.

6. Moreno, S. G.; Tornell, A. P.; Moreno, J.; At. Spectrosc. 1999, 20, 113

7. Gallay, W.; Egan, H.; Environmental Pollutants - Selected Analytical Methods, Butterworths \& Co: London, 1975.

8. NIOSH, National Institute for Occupational Safety and Health; Manual of Analytical Methods, $4^{\text {th }}$ ed., Public Health Service Centers for Disease Control and Prevention, Cincinnati, 1994.

9. Quintal, M. H. B.; Cano, J. A. M.; Salas, E. O. R.; Rodríguez, J. F.; Anal. Lett. 2001, 34, 2349.

10. Nimmo, M.; Fones, G.; Anal. Chim. Acta 1994, 291, 321.

11. Wang, J.; Stripping Analysis - Principles, Instrumentation and Application, VCH: New York, 1985.

12. Souza, D.; Machado, S. A. S.; Avaca, L .A.; Quim. Nova 2003, 26, 81.

13. Filipe, O. M. S.; Brett, C. M. A.; Talanta 2003, 61, 643.

14. Di, J.; Zhang, F.; Talanta 2003, 60, 31.

15. Viltchinskaia, E. A.; Zeigman, D. M.; Garcia, D. M.; Santos, P. F.; Anal. Lett. 1995, 28, 1845.

16. Labuda, J.; Vanícková, M.; Beinrohr, E.; Mikrochim. Acta 1989, I, 113.

17. Pedrosa, V. D.; Codognoto, L.; Machado, S. A. S.; Avaca, L.A.; J. Electroanal. Chem. 2004, 573, 11.

18. Pedrosa, V. D.; Codognoto, L.; Avaca, L. A.; Quim. Nova 2003, $26,844$.

19. Suffredini, H. B.; Machado S. A. S.; Avaca, L. A.; J. Braz. Chem. Soc. 2004, 15, 16.

20. Pedrosa, V. D.; Codognoto, L.; Avaca, L. A.; J. Braz. Chem. Soc. 2003, 14, 530 .

21. Peilin, Z.; Jianzhong, Z.; Shenzhong, Y.; Xikang, Z.; Guoxiong, Z.; Fresenius J. Anal. Chem. 1995, 353, 171.

22. Alehashem, S.; Chambers, F.; Strojek, J. W.; Swain, G. M.; Anal. Chem. 1995, 67, 2812.

23. Xu, J.; Chen, Q.; Swain, G. M.; Anal. Chem. 1998, 70, 3146.

24. Spear, K. E.; Dismukes, J. P.; Synthetic Diamond-Emerging CVD Science and Techonology, John Wiley \& Sons, New York, 1994.

25. Ferreira, N. G.; Silva, L. L. G.; Corat, E. J.; Trava-Airoldi, V. J.; Diamond Relat. Mater. 2002, 11, 1523.

26. Rodrigues, S.; Munichandraiah, N.; Shukla, A. K.; J. Appl. Electrochem. 1998, 28, 1235.

27. Petitpierre, P.; Plattner, C. C. E.; Electrochim. Acta 1990, 35, 281.

28. Davies, G.; Coord. Chem. Rev. 1969, 4, 199 
29. Paul, R. L.; Cartwright, A.; J. Electroanal. Chem. 1986, 201, 113.

30. Paul, R. L.; Cartwright, A.; J. Electroanal. Chem. 1986, 201, 123.

31. Kao, W. H.; Weibel, V. J.; J. Appl. Electrochem. 1992, 22, 21.

32. Bodoardo, S.; Brenet, J.; Maja, M.; Spinelli, P.; Electrochim. Acta 1994, 39, 1999.

33. Ruetschi, P.; J. Electrochem. Soc. 1984, 131, 2737.

34. Ruetschi, P.; J. Electrochem. Soc. 1988, 135, 2657.

35. Saterlay, A. J.; Foord, J. S.; Compton, R. G.; Analyst 1999, 124, 1791.

36. Lee, J. A.; Maskell, W. C.; Tye, F. L.; J. Electroanal. Chem. 1977, 79, 79.
37. Lee, J. A.; Maskell, W. C.; Tye, F. L.; J. Electroanal. Chem. 1980, 110, 145.

38. Miller, J. C.; Miller, J. N.; Statistics for Analytical Chemistry, $2^{\text {nd }}$ ed., John Wiley \& Sons: New York, 1988.

39. Felix, F. S.; Dissertação de Mestrado, Universidade de São Paulo, Brasil, 2004.

40. Felix, F. S.; Lichtig, J.; dos Santos, L. B. O.; Masini, J. C.; J. Braz. Chem. Soc., 2005, 16, 80

41. Jin, J. Y.; Xu, F.; Miwa, T.; Electroanal. 2000, 12, 610. 